\title{
Prognostic Effect of Inflammatory Genes on Stage I-III Colorectal Cancer-Integrative Analysis of TCGA Data
}

\author{
Eun Kyung Choe ${ }^{1,2,3,+}$, Sangwoo Lee ${ }^{4,+}$, So Yeon Kim ${ }^{2,5}$, Manu Shivakumar ${ }^{2}$, Kyu Joo Park ${ }^{3}$, Young Jun Chai ${ }^{6}$ \\ and Dokyoon Kim ${ }^{2,7, *}$
}

1 Department of Surgery, Seoul National University Hospital Healthcare System Gangnam Center, Seoul 06236, Korea; choe523@snu.ac.kr

2 Department of Biostatistics, Epidemiology \& Informatics, Perelman School of Medicine, University of Pennsylvania, Philadelphia, PA 19104-6116, USA; jebi1771@ajou.ac.kr (S.Y.K.); Manu.Shivakumar@pennmedicine.upenn.edu (M.S.)

3 Department of Surgery, Seoul National University College of Medicine, Seoul 03080, Korea; kjparkmd@snu.ac.kr

4 Department of Future Convergence, Cyber University of Korea, Seoul 03051, Korea; sangwoo07lee@cuk.edu

5 Department of Software and Computer Engineering, Ajou University, Suwon 16499, Korea

6 Department of Surgery, Seoul Metropolitan Government-Seoul National University Boramae Medical Center, Seoul 07061, Korea; kevinjoon@snu.ac.kr

7 Institute for Biomedical Informatics, University of Pennsylvania, Philadelphia, PA 19104-6116, USA

* Correspondence: dokyoon.kim@pennmedicine.upenn.edu; Tel.: +1-215-573-5336

+ Eun Kyung Choe and Sangwoo Lee contributed equally to the writing of this article.

\section{check for} updates

Citation: Choe, E.K.; Lee, S.; Kim, S.Y.; Shivakumar, M.; Park, K.J.; Chai, Y.J.; Kim, D. Prognostic Effect of Inflammatory Genes on Stage I-III Colorectal Cancer-Integrative Analysis of TCGA Data. Cancers 2021, 13, 751. https://doi.org/10.3390/ cancers13040751

Academic Editors:

Damián García-Olmo and Alessandro Cama

Received: 14 December 2020

Accepted: 7 February 2021

Published: 11 February 2021

Publisher's Note: MDPI stays neutral with regard to jurisdictional claims in published maps and institutional affiliations.

Copyright: (c) 2021 by the authors. Licensee MDPI, Basel, Switzerland. This article is an open access article distributed under the terms and conditions of the Creative Commons Attribution (CC BY) license (https:// creativecommons.org/licenses/by/ $4.0 /)$.
Simple Summary: Research interest in the role of inflammation in the progression and prognosis of colorectal cancer (CRC) is growing. In this study, we evaluated the expression and DNA methylation levels of inflammation-related genes in CRC tissues using the TCGA-COREAD dataset by integratively combining multi-omics features using machine learning. Statistical analysis was additionally performed to allow for interpretable, understandable, and clinically practical results. An integrative model combining expression, methylation, and clinical features had the highest performance. In multivariate analysis, the methylation levels of CEP250, RAB21, and TNPO3 were significantly associated with overall survival. Our study results implicate the importance of integrating expression and methylation information along with clinical information in the prediction of survival. CEP250, RAB21, and TNPO3 in the prediction model might have a crucial role in CRC prognosis and further improve our understanding of potential mechanisms linking inflammatory reactions and CRC progression.

Abstract: Background inflammatory status indicators have been reported as prognostic biomarkers of colorectal cancer (CRC). However, since inflammatory interactions with the colon involve various modes of action, the biological mechanism linking inflammation and CRC prognosis has not been fully elucidated. We comprehensively evaluated the predictive roles of the expression and methylation levels of inflammation-related genes for CRC prognosis and their pathophysiological associations. Method. An integrative analysis of 247 patients with stage I-III CRC from The Cancer Genome Atlas was conducted. Lasso-penalized Cox proportional hazards regression (Lasso-Cox) and statistical Cox proportional hazard regression $(\mathrm{CPH})$ were used for the analysis. Results. Models to predict overall survival were designed with respective combinations of clinical variables, including age, sex, stage, gene expression, and methylation. An integrative model combining expression, methylation, and clinical features performed better (median C-index $=0.756$ ) than the model with clinical features alone (median $\mathrm{C}$-index $=0.726$ ). Based on multivariate $\mathrm{CPH}$ with features from the best model, the methylation levels of CEP250, RAB21, and TNPO3 were significantly associated with overall survival. They did not share any biological process in functional networks. The 5-year survival rate was $29.8 \%$ in the low methylation group of CEP250 and 79.1\% in the high methylation group $(p<0.001)$. Conclusion. Our study results implicate the importance of integrating expression and methylation information along with clinical information in the prediction of survival. CEP250, RAB21, and TNPO3 in the prediction model might have a crucial role in CRC prognosis and further improve our understanding of potential mechanisms linking inflammatory reactions and CRC progression. 
Keywords: colorectal cancer; inflammatory genes; multi-omics data; TCGA

\section{Introduction}

Oncologic research on the role of inflammation in the progression and prognosis of cancer is growing [1]. Candidate inflammatory biomarkers, such as neutrophils, lymphocytes, monocytes, platelets, the lymphocyte-to-monocyte ratio (LMR), the neutrophil-tolymphocyte ratio (NLR), and the platelet-to-lymphocyte ratio (PLR), have been suggested as predictive factors for cancer prognosis [2-4]. In the colorectum, molecular pathophysiological analyses imply that inflammation promotes the progression of colorectal cancer [5]. The role of inflammation in the colorectum is well manifested in inflammatory bowel diseases such as ulcerative colitis and Crohn's disease. In these patients, chronic inflammation is a major risk factor for the development of gastrointestinal malignancies, especially colorectal cancer (CRC) [6]. Inflammatory biomarkers such as NLR, LMR, and PLR have been suggested as prognostic markers in colorectal cancer [7-10]. Chronic inflammation can trigger genetic mutations and epigenetic alterations that promote malignant cell transformation [11]. However, inflammatory interactions with the colon involve various modes of action, such as immune cells, cytokines, and other immune mediators, in virtually all sequences of CRC progression, including initiation, progression, and metastasis [12]. In recent reports, it has been postulated that epigenetic changes have a critical role in establishing efficient expression profiles during inflammation and disease [13-15]. Due to the complexity of the association between inflammation and CRC, the underlying mechanism linking inflammation and CRC prognosis has not been fully elucidated. To elucidate this mechanism, integrative analysis of multi-omics data with a genome-wide view is necessary [16].

The Cancer Genome Atlas (TCGA) has a collection of genomic and epigenomic data along with the clinical data of patients for a large number of cancer types [17]. The TCGA aims to develop a catalog of major cancer genomics through integrated multidimensional analyses [18]. This goal has increasingly fueled the prediction of various oncological outcomes based on multi-omics data [19-22].

In this study, we systematically evaluated the expression and DNA methylation levels of inflammation-related genes in CRC tissues using the TCGA-COREAD dataset. We comprehensively analyzed the whole set of inflammation-related genes based on both RNA expression and methylation to evaluate the predictive roles of these genes in overall survival and examine their significance levels in association with overall survival. By using Lasso-penalized Cox proportional hazards regression (Lasso-Cox), features were selected to design the prediction model for overall survival and used to evaluate whether the genetic features could improve the prediction performance in addition to clinical prognostic factors such as age and TNM staging. To our knowledge, no previous studies have focused on the use of integrated omics biomarkers, particularly inflammation-related genes, as additive features to the basis of the current TNM staging system in designing a prediction model for overall survival in colorectal cancer.

\section{Methods}

\subsection{Data Acquisition and Preprocessing}

We utilized the clinical information, RNA-seq gene data, and DNA methylation profiles of The Cancer Genome Atlas-colorectal adenocarcinoma (TCGA-COREAD). Clinical information and RNA-seq gene expression were downloaded from the UCSC Xena browser (https: / xenabrowser.net (accessed on 4 May 2019)). Gene-level methylation data of TCGACOREAD were retrieved from the Broad Institute Firebrowse (http:/ / firebrowse.org (accessed on 4 May 2019)). The gene expression data were acquired using Illumina HiSeq 2000 RNA sequencing. The gene expression transcription estimates are shown as logtransformed RSEM normalized counts. For the RNA-seq data, genes that had more than 
$50 \%$ samples with 0 expression levels were removed. For the methylation data, we used the methylation data by picking the probe with the minimum correlation with mRNA-seq data for each gene. Genes that had zero methylation levels in all samples were excluded. Two authors (EKC, KJP) who are colorectal surgeons independently reviewed the pathological report and revised incorrect or inconsistent clinical information. Among the 736 samples in the data, we excluded the samples based on the clinical information as follows: samples collected from normal tissue; stage IV colorectal cancer; missing data on pathological stages, survival data, and age information; and follow-up duration less than 12 months. For clinical information, age, sex, TNM stage, overall survival, and event status were used as clinical variables. Finally, we chose the data for analysis using the overlapping sample IDs among the RNA-seq, methylation and clinical data sets. Thus, 247 patients were used for further analyses.

\subsection{Ethics Statement}

On TCGA publication guidelines (http: / / cancergenome.nih.gov/publications / publicationguidelines (accessed on 4 May 2019)), there are no restrictions on the publication or use of the dataset. The study was conducted in accordance with the Declaration of Helsinki.

\subsection{Feature Selection}

Figure 1 shows an overview of the analysis framework. First, we selected the gene sets related to the inflammatory status from the National Center for Biotechnology Information's (NCBI) gene database (www.ncbi.nlm.nih.gov/gene (accessed on 15 May 2019)) [23], with the filtering term "(inflammatory) AND "homo sapiens"[porgn:_txid9606]". A total of 2576 genes were retrieved and used as inflammation-related genes for the analyses. Then, we preprocessed the gene sets and the clinical information (such as age ( $<65$ years vs. $\geq 65$ years), sex (male vs. female), $\mathrm{T}$ stage (T1, T2 vs. T3, T4), and N stage (N0 vs. N1, N2) based on the significance of their association with overall survival determined by univariate Cox proportional hazard regression $(\mathrm{CPH})$ analysis. The clinical, expression, and methylation features that passed the suggestive significance level $(p<0.01)$ were used for further analyses (Clinical, Expression, Methylation). We also made an input matrix in which expression and methylation data were concatenated (Expression + Methylation) (Figure 1). Finally, feature selection was performed for the respective data sets (Clinical, Expression, Methylation, and Expression + Methylation) by using Lasso-penalized Cox proportional hazards regression (Lasso-Cox). The training set versus test set ratio was 8:2. We selected important features that showed the best prediction performance by training the Lasso-Cox model in the Clinical, Expression, Methylation, and Expression + Methylation dataset. To validate the feature selection model, we performed 50 iterations of 5-fold cross-validation in the training set.

\subsection{Overall Survival Prediction Based on Omic Features}

Using the selected features, we trained prediction models for overall survival using Lasso-penalized Cox proportional hazards regression and compared the performances of each model. Seven models were designed by combining features such as clinical features (C); Expression features (E); Methylation features (M); Expression + Methylation features (EM); Clinical features + Expression features (C-E); Clinical features + Methylation features (C-M); and Clinical features + Expression + Methylation features (C-EM). The performances among the models were compared by the concordance index (C-index), which measures how concordant the observations and predictions by our model are for a pair of randomly chosen patient samples [24] (Figure 1). After performing the analysis using machine learning, we tried to apply statistical analysis to the model to make the results interpretable and evident for clinical utility. Among the models, we used the model that achieved the highest $\mathrm{C}$-index to perform multivariate $\mathrm{CPH}$ analysis to examine which features had a significant association with overall survival $(p<0.05)$. Then, Kaplan-Meier analysis was 
carried out using the genes that were significantly associated with overall survival. The optimal cutoff points for those genes, to divide patients into two groups, were determined by MaxStat packages in R (maximally selected rank statistics). MaxStat performs a test of independence of response and one or more covariables using maximally selected rank statistics [25]. The log-rank test was used to compare the survival curves of the different gene-level groups. Kaplan-Meier plots were visualized by the "ggkm" R package. Finally, integrated multispecies prediction (IMP) [26] was used to visualize the gene-gene network and biological process network. IMP integrates multiple sources of evidence for functional interactions by integrating 3741 genome-scale datasets for 7 organisms and predicts 582 disease and 12,117 biological processes (http: / / imp.princeton.edu/ (accessed on 29 July 2019)) [27]. We queried IMP with the gene set from the best prediction model. All statistical and computational analyses were performed using $\mathrm{R}$ statistical software (version 3.5.3 R development Core Team; R Foundation for Statistical Computing, Vienna, Austria). The putative association between clinical information and survival outcome was assessed by Chi-square test, Student's t-test and analysis of variance (ANOVA) for independent groups in Table 1.

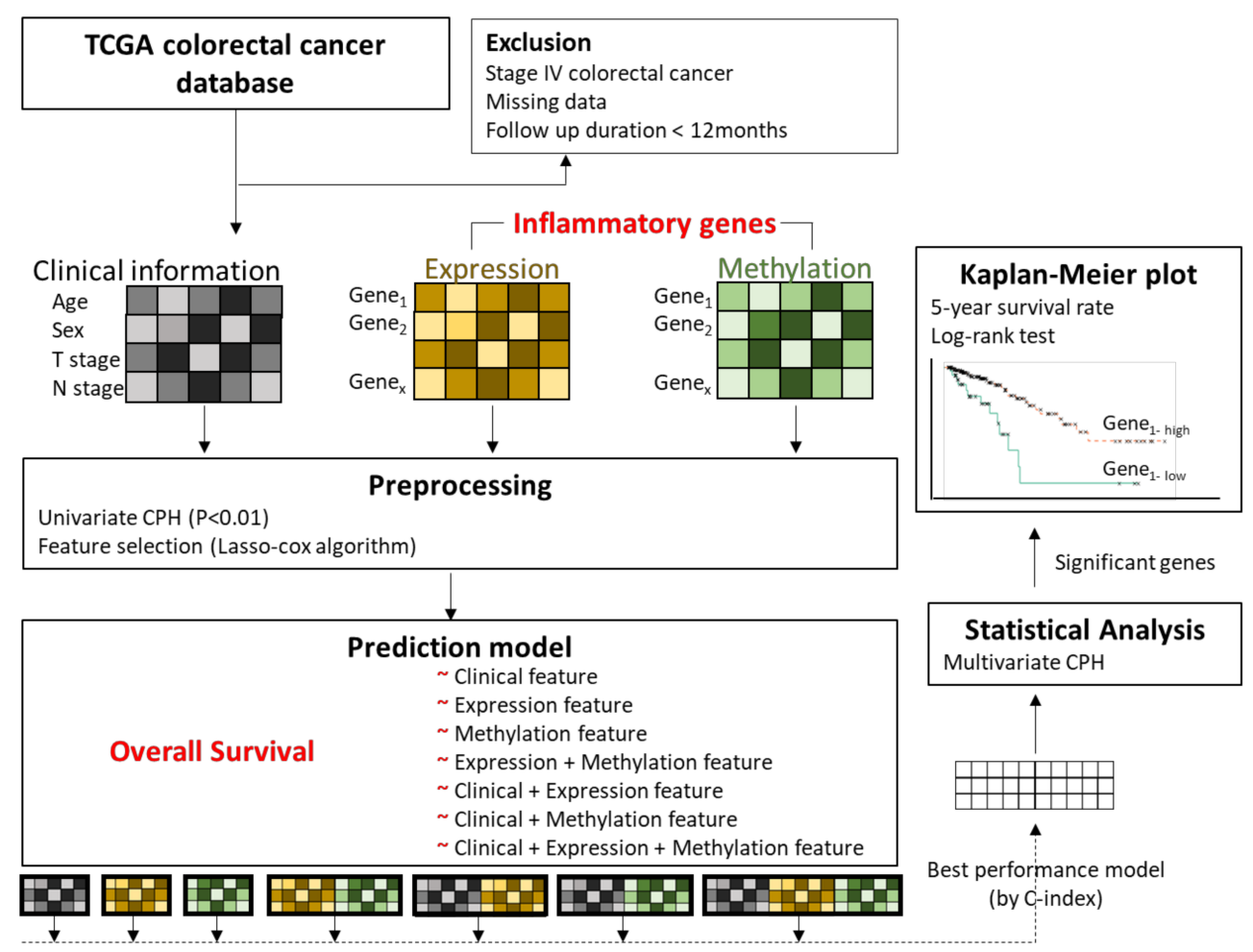

Figure 1. Overview of the analysis framework. We preprocessed the gene sets related to inflammatory status and clinical information, based on the significance of association with overall survival by performing univariate Cox proportional hazard regression $(\mathrm{CPH})$ analysis. These features passed the suggestive significance level $(p<0.01)$. Features with concatenated expression and methylation were also included. Feature selection was performed by using Lasso-penalized Cox proportional hazards regression (Lasso-Cox). Seven modes were designed by respective combinations of features, and the performances were evaluated by Lasso-Cox according to the C-index. Among the models, the highest C-index model was used for multivariate $\mathrm{CPH}$ analysis of overall survival. Then, Kaplan-Meier analysis was performed for the genes that were significantly associated with overall survival, and the log rank test was performed for comparisons of each group. 
Table 1. Demographic features of the study population.

\begin{tabular}{|c|c|c|c|}
\hline Features & Alive & Dead & $p$ Value \\
\hline Age & $62.9 \pm 12.5$ & $71.7 \pm 12.6$ & $<0.001$ \\
\hline$<65$ & $111(53.1 \%)$ & $7(18.4 \%)$ & \\
\hline$\geq 65$ & $98(46.9 \%)$ & $31(81.6 \%)$ & \\
\hline Continuous & $62.9 \pm 12.5$ & $71.7 \pm 12.6$ & \\
\hline Gender & & & 0.407 \\
\hline Male & $108(51.7 \%)$ & $23(60.5 \%)$ & \\
\hline Female & $101(48.3 \%)$ & $15(39.5 \%)$ & \\
\hline Overall stage & & & 0.201 \\
\hline Stage 1,2 & $136(65.1 \%)$ & $20(52.6 \%)$ & \\
\hline Stage 3 & $73(34.9 \%)$ & $18(47.4 \%)$ & \\
\hline Stage 1 & $46(22.0 \%)$ & $2(5.3 \%)$ & \\
\hline Stage 2 & $90(43.1 \%)$ & $18(47.4 \%)$ & \\
\hline Stage 3 & $73(34.9 \%)$ & $18(47.4 \%)$ & \\
\hline $\mathrm{T}$ stage & & & 0.046 \\
\hline $\mathrm{T} 1, \mathrm{~T} 2$ & $50(23.9 \%)$ & $3(7.9 \%)$ & \\
\hline $\mathrm{T} 3, \mathrm{~T} 4$ & $159(76.1 \%)$ & $35(92.1 \%)$ & \\
\hline $\mathrm{T} 1$ & $8(3.8 \%)$ & $1(2.6 \%)$ & \\
\hline $\mathrm{T} 2$ & $42(20.1 \%)$ & $2(5.3 \%)$ & \\
\hline T3 & $146(69.9 \%)$ & $32(84.2 \%)$ & \\
\hline $\mathrm{T} 4$ & $13(6.2 \%)$ & $3(7.9 \%)$ & \\
\hline N stage & & & 0.113 \\
\hline LN negative & $136(65.1 \%)$ & $19(50.0 \%)$ & \\
\hline LN positive & $73(34.9 \%)$ & $19(50.0 \%)$ & \\
\hline N0 & $136(65.1 \%)$ & $19(50.0 \%)$ & \\
\hline N1 & $49(23.4 \%)$ & $9(23.7 \%)$ & \\
\hline N2 & $24(11.5 \%)$ & $10(26.3 \%)$ & \\
\hline Tumor location & & & 1 \\
\hline Right colon & $102(50.2 \%)$ & $17(48.6 \%)$ & \\
\hline Left colon & $101(49.8 \%)$ & $18(51.4 \%)$ & \\
\hline Venous invasion & & & 0.916 \\
\hline Negative & 147 (80.8\%) & $25(78.1 \%)$ & \\
\hline Positive & $35(19.2 \%)$ & $7(21.9 \%)$ & \\
\hline Lymphatic invasion & & & 0.887 \\
\hline Negative & $140(75.7 \%)$ & $24(72.7 \%)$ & \\
\hline Positive & $45(24.3 \%)$ & $9(27.3 \%)$ & \\
\hline Follow up duration (months) & $38.54 \pm 31.26$ & $39.97 \pm 22.79$ & 0.739 \\
\hline
\end{tabular}

\section{Results}

\subsection{Patient Demographics}

Our study population comprised 247 patients (131 males and 116 females) who underwent colectomy for stage I-III colorectal cancer. The mean patient age was $64.28 \pm 12.90$ years, and the median follow-up was 26.83 months (ranging from 12.17 to 150.07 months). Patients' characteristics are shown in Table 1.

\subsection{Feature Selection for Overall Survival with Lasso-Cox}

We preprocessed the gene sets and the clinical information based on the significance of association with overall survival by performing univariate $\mathrm{CPH}$ analysis. Features that passed the suggestive significance level $(p<0.01)$ were 4 clinical variables (age, sex, T stage, and $\mathrm{N}$ stage), 26 gene expression and 14 methylation features. By concatenating the selected expression and methylation features, Expression + Methylation data had 40 features. After feature selection using Lasso-Cox, 4 variables were selected for clinical features (C), 16 for expression features (E), 12 for methylation features (M), and 14 for omic features from the combined dataset (EM). The list of the features is shown in Table S1. 


\subsection{Training Prediction Models for Overall Survival with Lasso-Cox}

Using various combinations from 4 feature sets (C, E, M, and EM), we designed seven models to predict overall survival (Figure 1). The performances of each model are shown in Table 2. Among the 4 respective feature sets, a model with EM showed the highest performance (median C-index $=0.727$ ). In particular, among models incorporating the genetic features into clinical features, an integrative model with C-EM showed the best performance (median C-index $=0.756$ ) (Table 2). Adding the respective $\mathrm{E}$ or $\mathrm{M}$ to $\mathrm{C}$ did not improve the performance compared to the model with $\mathrm{C}$ alone; however, integrating the $\mathrm{EM}$ and $\mathrm{C}$ showed great improvement in our prediction model.

Table 2. The prediction performances of each model were compared by the concordance index.

\begin{tabular}{ccccccc}
\hline Features Used & Min. & 1st Qu. & Median & Mean & 3rd Qu. & Max. \\
\hline Clinical features & 0.318 & 0.648 & 0.726 & 0.706 & 0.791 & 0.921 \\
\hline Expression features & 0.449 & 0.614 & 0.691 & 0.688 & 0.774 & 0.899 \\
\hline Methylation features & 0.447 & 0.580 & 0.686 & 0.683 & 0.772 & 0.899 \\
\hline $\begin{array}{c}\text { Expression features } \\
+ \text { Methylation features }\end{array}$ & 0.337 & 0.647 & 0.727 & 0.715 & 0.826 & 0.884 \\
\hline $\begin{array}{c}\text { Clinical features } \\
+ \text { Expression features }\end{array}$ & 0.438 & 0.609 & 0.667 & 0.673 & 0.761 & 0.832 \\
\hline $\begin{array}{c}\text { Clinical features } \\
+ \text { Methylation features }\end{array}$ & 0.333 & 0.628 & 0.704 & 0.682 & 0.757 & 0.866 \\
\hline $\begin{array}{c}\text { Clinical features } \\
+ \text { Expression features } \\
+ \text { Methylation features }\end{array}$ & 0.326 & 0.655 & 0.756 & 0.708 & 0.818 & 0.883 \\
\hline
\end{tabular}

\subsection{Association Analysis of Overall Survival with Multivariate Cox Proportional} Hazard Regression

For the model with C-EM, the best prediction model, an association study was performed to examine the association between the selected features and overall survival. By multivariate CPH, CEP250 ( $p=0.035), \operatorname{RAB} 21(p=0.002)$, and TNPO3 $(p=0.011)$ as methylation features showed independent associations with overall survival (Table 3). For the three methylation features, Kaplan-Meier plots were generated (Figure 2). The patients were divided by the optimal cutoffs for each feature based on MaxStat $(-0.923$ for CEP250; 0.5 for TNPO3, and -0.367 for RAB21). Figure 2 shows the Kaplan-Meier plots with the results of the log-rank test. The 5-year survival rate of TNPO3 was $81.1 \%$ for methylation below the cutoff point and $76.5 \%$ for methylation above the cutoff point; for RAB21, the rate was $54.4 \%$ for methylation below the cutoff point and $76.5 \%$ for methylation above the cutoff point; and for CEP250, the rate was $29.8 \%$ for methylation below the cutoff point and $79.1 \%$ for methylation above the cutoff point.

\subsection{Gene-Gene Network and Biological Process Network}

We visualized the functional network of the expression and methylation features used in the prediction model C-EM using IMP. For gene-gene networking construction, we queried all 14 genes used in the model, and the results are shown in Figure 3a. Except for RAB21, TERF21P, and PPARGC1A, 11 genes among 14 genes had less than 3 strong relationships with other genes in the network. The biological process network for the 3 genes, which are significantly associated with overall survival, was also visualized (Figure $3 \mathrm{~b}$ ). We used a confidence level of 0.5 when visualizing networking. Notably, all genes had unique biological processes, and there were no shared biological processes among the three genes. 
Table 3. Association analysis of the integrative model with clinical, gene expression and methylation features and overall survival. Analysis was performed with multivariate Cox proportional hazard regression. CEP250, centrosomal protein 250; DEFA5, defensin alpha 5; MAZ, MYC-associated zinc finger protein; NINJ, nerve injury-induced protein 1; NLRP14 (expression), NLR family pyrin domain-containing 14; PPARGC1A, peroxisome proliferator-activated receptor $\gamma$ coactivator 1 alpha; PRG4, proteoglycan 4; PTGES, prostaglandin E synthase; RAB21, member of the RAS oncogene family; TERF2IP, TERF2-interacting protein; TMEM184A, transmembrane protein 184A; TNFRSF18, TNF receptor superfamily member 18; TNFSF12 TNF superfamily member 12; TNPO3, transportin 3.

\begin{tabular}{cccccc}
\hline Features & $\begin{array}{c}\text { Hazard } \\
\text { Ratio (HR) }\end{array}$ & $\begin{array}{c}\text { 95\% CI, } \\
\text { Lower }\end{array}$ & $\begin{array}{c}\mathbf{9 5 \%} \text { CI, } \\
\text { Upper }\end{array}$ & Z Value & $\begin{array}{c}\text { Adjusted } \\
\boldsymbol{p} \text { Value }\end{array}$ \\
\hline Age & 1.697 & 0.609 & 4.733 & 1.011 & 0.312 \\
\hline N stage & 2.942 & 1.253 & 6.912 & 2.477 & 0.013 \\
\hline T stage & 0.919 & 0.237 & 3.557 & -0.122 & 0.903 \\
\hline Gender & 0.910 & 0.390 & 2.121 & -0.219 & 0.827 \\
\hline CEP250 (methylation) & 0.592 & 0.364 & 0.963 & -2.110 & 0.035 \\
\hline DEFA5 (expression) & 0.786 & 0.462 & 1.337 & -0.888 & 0.374 \\
\hline MAZ (methylation) & 0.967 & 0.809 & 1.156 & -0.369 & 0.712 \\
\hline NINJ1 (methylation) & 1.339 & 0.910 & 1.968 & 1.482 & 0.138 \\
\hline NLRP14 (expression) & 0.797 & 0.525 & 1.211 & -1.063 & 0.288 \\
\hline $\begin{array}{c}\text { PPARGC1A } \\
\text { (expression) }\end{array}$ & 0.808 & 0.636 & 1.027 & -1.744 & 0.081 \\
\hline PRG4 (expression) & 1.256 & 0.871 & 1.811 & 1.219 & 0.223 \\
\hline PTGES (expression) & 1.399 & 0.937 & 2.087 & 1.644 & 0.100 \\
\hline RAB21 (methylation) & 1.556 & 1.172 & 2.065 & 3.060 & 0.002 \\
\hline TERF2IP (expression) & 1.452 & 0.893 & 2.360 & 1.502 & 0.133 \\
\hline $\begin{array}{c}\text { TMEM184A } \\
\text { (expression) }\end{array}$ & 1.251 & 0.819 & 1.911 & 1.038 & 0.299 \\
\hline $\begin{array}{c}\text { TNFRSF18 } \\
\text { (methylation) }\end{array}$ & 1.489 & 0.783 & 2.829 & 1.214 & 0.225 \\
\hline $\begin{array}{c}\text { TNFSF12 } \\
\text { (methylation) }\end{array}$ & 1.132 & 0.801 & 1.600 & 0.704 & 0.481 \\
\hline TNPO3 (methylation) & 1.465 & 1.092 & 1.967 & 2.543 & 0.011 \\
\hline & & & & & \\
\hline
\end{tabular}



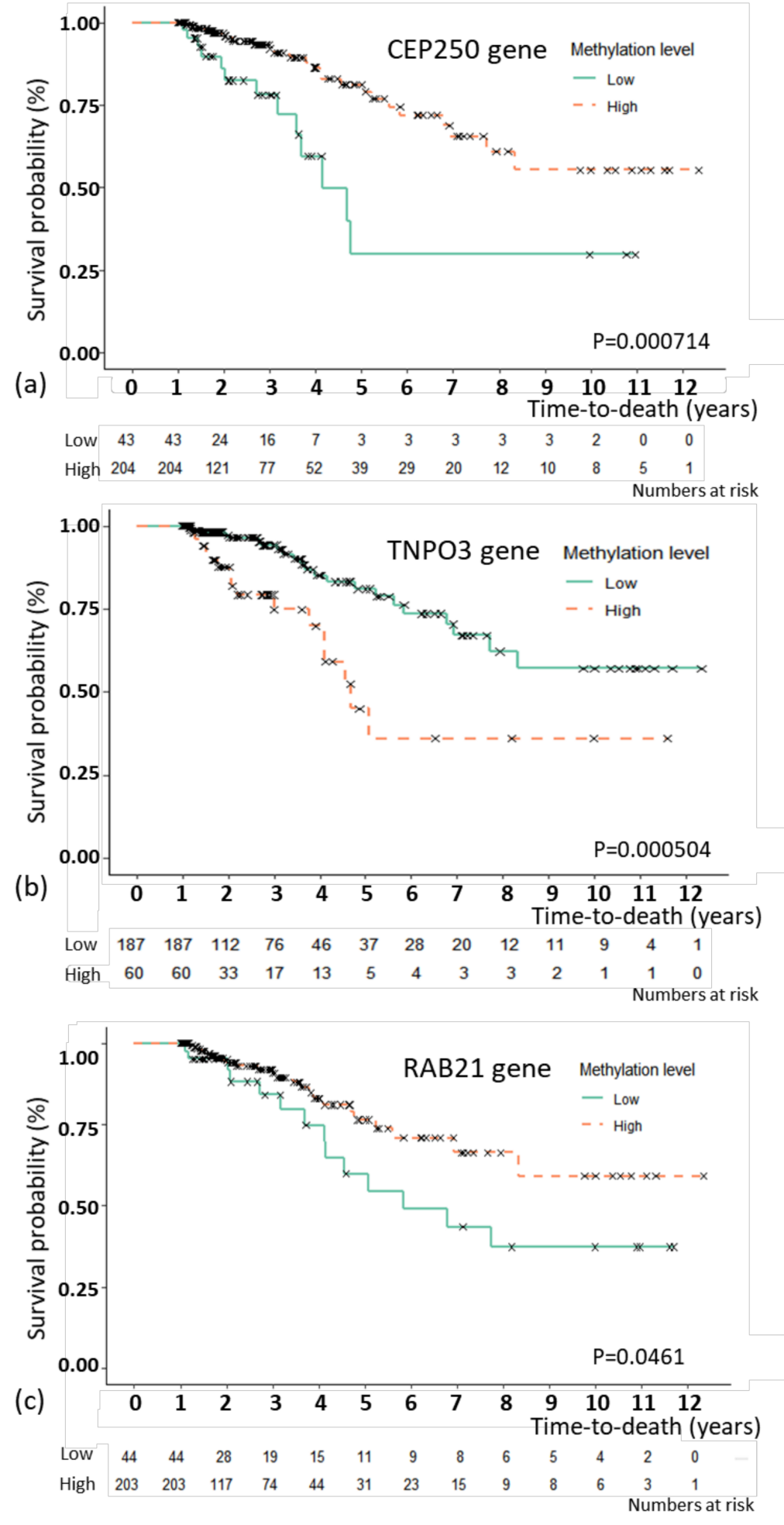

Figure 2. Kaplan-Meier plot of overall survival with genes that were significant in the multivariate Cox proportional hazard regression analysis. Kaplan-Meier plot of overall survival with the CEP250 (a), TNPO3 (b), and RAB21 (c) genes. The patients were divided by the optimal cutoffs for each feature based on MaxStat ( -0.923 for CEP250; 0.5 for TNPO3, and -0.367 for RAB21). 


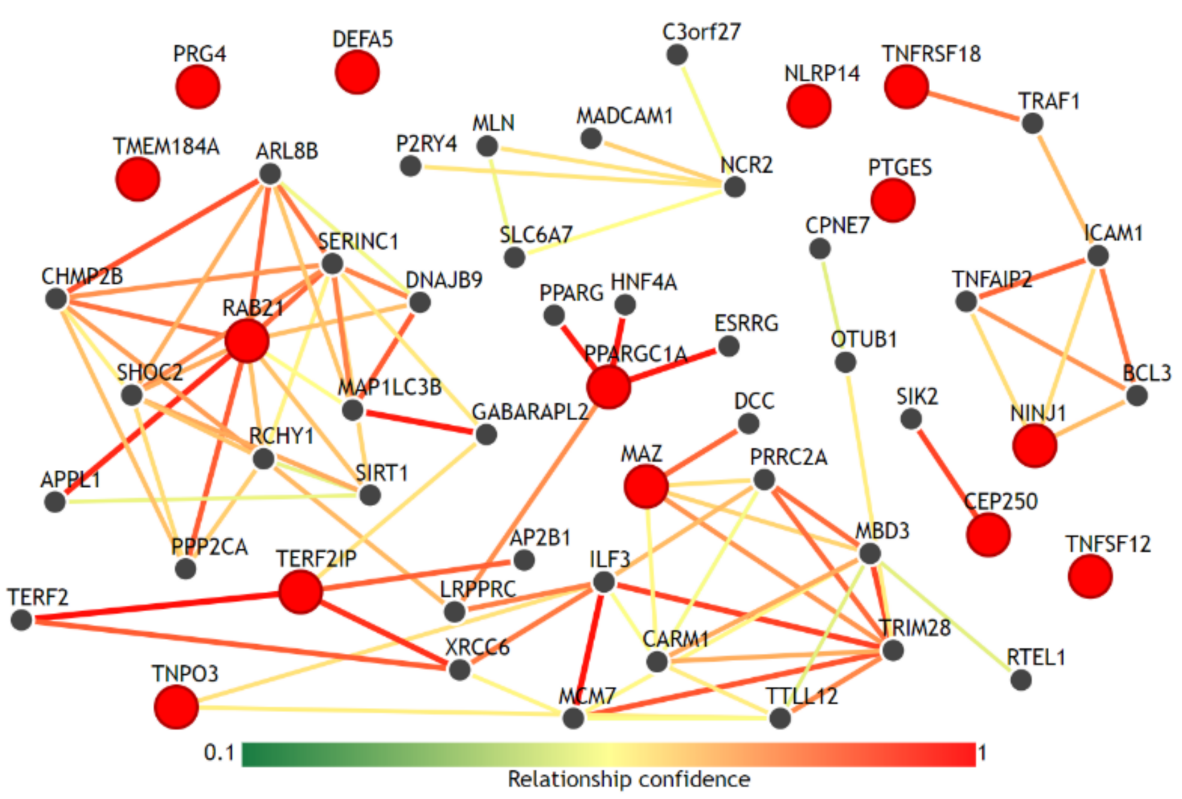

(a)

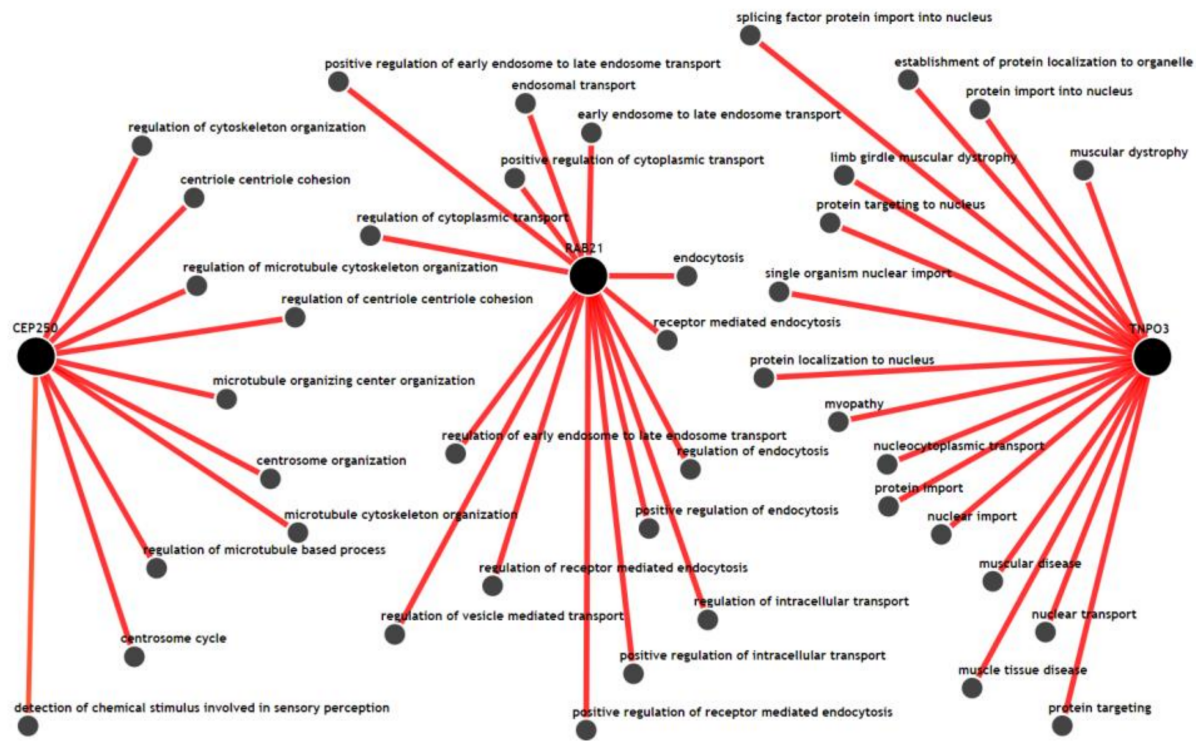

(b)

Figure 3. Functional network of the gene features used in the integrative model with clinical, gene expression and methylation features for the prediction of overall survival. (a) Gene-gene networks queried by 14 genes in the model. Red dots are the queried genes (14 genes, Table S1), and the black dots are the genes predicted based on functional networking analysis ( 0.5 confidence with 40 genes). Except for RAB21, TERF21P, and PPARGC1A, most of the genes among the 14 genes had fewer than 3 strong relationships with other genes by network. (b) Biological process networks queried by 3 genes (CEP250, TNPO3, and RAB21) that showed a significant association with overall survival (confidence 0.5). All genes had unique biological processes, and there were no shared biological processes among the three genes.

\section{Discussion}

Age, sex, T stage, and N stage, which are used in this study as clinical features, are the most commonly used clinical factors for predicting the prognosis and survival of patients with colorectal cancer [28]. Comparing the prediction performances of these clinical factors, the integrative model that integrated clinical, expression and methylation 
features significantly improved the performance of predicting overall survival. Adding expression features alone or methylation features alone to the clinical features did not contribute to the improvement in performance. However, only the combined dataset between expression and methylation features was able to improve the performance. This finding might reflect the fact that inflammatory interactions with CRC include a wide range of biological processes in immune cells, cytokines, and other immune mediators in virtually all sequences of CRC progression, including initiation, progression, and metastasis [12]. This result is also consistent with our results using IMP. In the gene-gene network (Figure $3 a)$, the selected genes in the prediction model did not have high-dimensional networks among each other, except for RAB21 and PPARGC1A, and all genes had fewer or no connections to other genes. This could imply that these genes uniquely contribute to the inflammation-cancer progression mechanism. In addition, in the biological process network (Figure 3b), the genes associated significantly with overall survival (RAB21, CEP250, and TNPO3) did not share any biological processes. This seems to be in accordance with the finding that they were statistically independent factors for association with overall survival.

In the best integrative prediction model, 7 expression and 7 methylation features were selected. We performed a literature review on the function of these gene features and their relationship with inflammation. The CEP250 gene and TERF2IP gene are involved in the cell cycle. The CEP250 gene encodes a centrosomal protein contributing to centrosomecentrosome cohesion during interphase of the cell cycle [29]. It has been reported to be associated with inflammation in joints and bones [30]. There is also a genome-wide association study that shows the association of CEP250 with inflammatory bowel disease [31]. TERF21P encodes a protein involved in telomere length regulation [29]. Several studies have shown that their aberrant activation leads to increased cancer cell proliferation and tumorigenesis [32,33].

The RAB21 gene and NINJ1 gene are related to cell adhesion and migration. The RAB21 gene is a Rab family of monomeric GTPases, and the encoded protein is related to the regulation of cell adhesion and migration [23]. It is involved in the inflammatory reaction by regulating lipopolysaccharide-induced proinflammatory responses [34]. It has been reported to be related to cancer invasion and cancer mobility [35,36]. The NINJ1 gene produces a protein that is a homophilic cell adhesion molecule [37] and plays a role in the progression of multiple sclerosis [38]. NINJ1 is a target of p53 and has been known to repress WT p53 expression [39].

The TNPO3, MAZ, and PPARGC1A genes are related to gene regulation. The TNPO3 gene encodes a nuclear import receptor for serine/arginine-rich (SR) proteins [29]. This gene is reported to be associated with several inflammatory autoimmune diseases, such as systemic lupus erythematosus [40] and rheumatoid arthritis [41]. There are no reports related to colon disease or cancer. The MAZ gene affects the expression of MYC gene [42]. The MYC gene is well known for its role in the progression of CRC [43]. The MYC gene is frequently deregulated in inflammation, and its expression is affected by DNA methylation [11]. The PPARGC1A gene encodes a transcriptional coactivator that regulates the genes involved in energy metabolism [29]. In an experiment, it was profoundly reduced in ulcerative colitis patients [44].

There are several genes related to cytokine reactions. The TNFRSF18 and TNFSF12 genes are related to tumor necrosis factor (TNF), which is a well-known inflammatory biomarker that promotes cytokines in colon cancer [12]. The NLRP14 gene encodes the NLRP (nucleotide-binding oligomerization domain, leucine-rich repeat and pyrin domain containing) protein family, which belongs to the inflammasome and activates proinflammatory enzymes [29]. The NLRP inflammasome is suggested as a possible linkage mechanism between obesity-associated low-grade chronic inflammation and CRC development [45]. The PTGES gene encodes a glutathione-dependent prostaglandin E synthase, and its expression seems to be induced by the proinflammatory cytokine interleukin 1 beta (IL1B) [29]. PTGES is upregulated in CRC and premalignant lesions such as colonic adenomatous polyps [46,47]. The DEFA5 gene encodes a family of antimicrobial and cytotoxic pep- 
tides thought to be involved in host defense [29]. It is reported as a candidate biomarker for Crohn's disease [48]. The PRG4 gene encodes a large proteoglycan [29]. It plays an important anti-inflammatory role in osteoarthritis synoviocyte proliferation [49]. In addition, PRG4 has been demonstrated to have anti-inflammatory properties [50], and it may suppress breast cancer cell invasion [51]. There are no reports related to colon disease or cancer.

For the TMEM184A gene, there are few reports on its pathophysiology in inflammation and cancer progression. A few reports have shown that TMEM 184A functions as a heparin receptor and regulates the anti-inflammatory response of endothelial cells [52].

As seen in the literature review, the selected inflammatory genes have a variety of pathophysiological mechanisms that could be considered to contribute to inflammationCRC linkage mechanisms. These genes could be the crucial sets of genes that could cover the majority of the mechanism linking inflammation-CRC progression. In addition, it could be postulated that the role of inflammatory genes in CRC progression is not related or connected to one functional pathway but is affected through multiple global mechanisms by respective inflammatory genes.

This study has several advantages. We comprehensively analyzed all genes related to inflammation to see the global network between inflammation and CRC progression and included both expression and methylation data for those genes. By combining the expression and methylation data in the model design, we were able to obtain a survival prediction model that improved the performance of conventional clinical prediction models. This performance would not be achievable if the model were to be designed using either gene expression or methylation data. Second, we performed both the prediction model based on computational analysis and the association study based on statistical analysis. This will help to interpret the results more intuitively and support evidence for the results of the prediction model.

However, there are a couple of limitations in our analysis. First, as we used an open-source database, the samples were not collected under well-controlled and unified conditions. The resulting heterogeneity of the samples could have made the characteristics of the population different from the real world population of CRC patients. We compared the 5-year overall survival rate in respective pathologic stages from our study population to that of colorectal cancer patients from the Surveillance, Epidemiology, and End Results (SEER) program, which is a large-scale data set from the United States [53]. The patients from our TCGA data set showed that patients with stage I, II, and III colorectal cancer had 5 -year overall survival rate of $83.1 \%, 80.3 \%$, and $55.0 \%$, respectively, as compared with $82.7 \%, 70.3 \%$, and $68.7 \%$ for those from the SEER program [54]. The 5-year overall survival rate in stage I was similar between two data sets. In stages II and III, which the prognosis is highly affected by various clinical factors other than overall stage, there were notable differences in the 5-year overall survival rates between two data sets. In the TCGA data set, which is an open-source database, there were many missing data. This prohibited us from using important clinical factors such as adjuvant therapy protocol, microsatellite instability, lymphatic invasion, and venous invasion for survival analysis. These clinical factors could also have a crucial role in predicting overall survival. The limitation of information on the study population characteristics might have limited the extent to which our study reflects the findings in the real world population with colorectal cancer. Second, we simply concatenated the clinical factors, RNA expression and methylation levels for integration. Transformational integration could be applied in a larger set of samples. Third, since there are no open-source databases that include both RNA expression and methylation data with long-term survival information, we could not validate the results in another data set. This should be performed in future studies by constructing a colorectal cancer cohort with multi-omics profiles and long-term outcome information. 


\section{Conclusions}

By analyzing colorectal cancer patients from TCGA datasets, we were able to develop prediction models for overall survival based on genetic profiles in relation to the inflammatory status. Overall survival was best predicted by the model combining expression and methylation features with clinical features, which outperformed the model designed using clinical features alone. This emphasizes the importance of introducing integrative omics biomarkers of the inflammation status as additive features to the current TNM staging system to improve prognostic predictions in patients with colorectal cancer. Functionally, the gene features of this model rarely share a functional network, implying that the inflammatory status contributes to colorectal cancer progression, not in a simple biological process but rather a global gene set linkage of biological processes. By association analysis, we were able to identify that the methylation levels of the CEP250, RAB21, and TNPO3 genes independently play crucial roles in colorectal cancer prognosis. These findings could be used to elucidate the underlying mechanism between the inflammatory status and colorectal cancer progression. We believe that the collection of various types of omics data from colorectal cancer patients in future studies will enable a better understanding of the pathophysiology of its disease mechanism.

Supplementary Materials: The following are available online at https:/ /www.mdpi.com/2072-669 4/13/4/751/s1, Table S1. Selected features by Lasso-Cox feature selection.

Author Contributions: Conceptualization, E.K.C., S.L., K.J.P., Y.J.C., and D.K.; methodology, E.K.C., S.L., and S.Y.K.; software, S.L. and M.S.; data curation, E.K.C. and S.L.; writing-original draft preparation, E.K.C., S.Y.K., and S.L.; writing—review and editing, E.K.C., M.S., K.J.P., Y.J.C., S.Y.K., S.L., and D.K.; visualization, E.K.C.; supervision, D.K. All authors have read and agreed to the published version of the manuscript.

Funding: This work was supported by the National Library of Medicine (NLM) R01 NL012535.

Institutional Review Board Statement: Ethical review and approval were waived for this study due to TCGA publication guidelines (http:/ / cancergenome.nih.gov/publications/publicationguidelines (accessed on 4 May 2019)); there are no restrictions on the publication of these data, and no specific permission is required for investigators to publish papers containing or referring to these data.

Informed Consent Statement: Patient consent was waived due to TCGA publication guidelines; there are no restrictions on the publication of these data, and no specific permission is required for investigators to publish papers containing or referring to these data.

Data Availability Statement: Clinical and RNA-seq data from The Cancer Genome Atlas-colorectal adenocarcinoma (TCGA-COREAD) are available from the UCSC Xena browser (https: / / xenabrowser. net). Gene-level methylation data from TCGA-COREAD are available from the Broad Institute Firebrowse (http: / firebrowse.org (accessed on 4 May 2019)).

Conflicts of Interest: The authors declare that they have no conflict of interest.

\section{Abbreviations}

$\begin{array}{ll}\text { CRC } & \text { Colorectal } \\ \text { TCGA-COREAD } & \text { The Cancer Genome Atlas-Colorectal Cancer } \\ \text { Lasso-Cox } & \text { Lasso-penalized Cox proportional hazards regression } \\ \text { CPH } & \text { Cox proportional hazard regression } \\ \text { IMP } & \text { Integrative multispecies prediction } \\ \text { LMR } & \text { Lymphocyte-to-monocyte ratio } \\ \text { NLR } & \text { Neutrophil-to-lymphocyte ratio } \\ \text { PLR } & \text { Platelet-to-lymphocyte ratio } \\ \text { C } & \text { Clinical features } \\ \text { E } & \text { Gene expression features } \\ \text { M } & \text { Gene methylation features } \\ \text { EM } & \text { Concatenated expression and methylation features }\end{array}$




\section{References}

1. Mantovani, A.; Allavena, P.; Sica, A.; Balkwill, F. Cancer-related inflammation. Nature 2008, 454, 436-444. [CrossRef]

2. Sun, K.; Chen, S.; Xu, J.; Li, G.; He, Y. The prognostic significance of the prognostic nutritional index in cancer: A systematic review and meta-analysis. J. Cancer Res. Clin. Oncol. 2014, 140, 1537-1549. [CrossRef]

3. Templeton, A.J.; Ace, O.; McNamara, M.G.; Al-Mubarak, M.; Vera-Badillo, F.E.; Hermanns, T.; Seruga, B.; Ocana, A.; Tannock, I.F.; Amir, E. Prognostic role of platelet to lymphocyte ratio in solid tumors: A systematic review and meta-analysis. Cancer Epidemiol. Biomark. Prev. 2014, 23, 1204-1212. [CrossRef]

4. Gu, L.; Li, H.; Chen, L.; Ma, X.; Li, X.; Gao, Y.; Zhang, Y.; Xie, Y.; Zhang, X. Prognostic role of lymphocyte to monocyte ratio for patients with cancer: Evidence from a systematic review and meta-analysis. Oncotarget 2016, 7, 31926-31942. [CrossRef] [PubMed]

5. Janakiram, N.B.; Rao, C.V. The role of inflammation in colon cancer. Adv. Exp. Med. Biol. 2014, 816, 25-52. [CrossRef] [PubMed]

6. Axelrad, J.E.; Lichtiger, S.; Yajnik, V. Inflammatory bowel disease and cancer: The role of inflammation, immunosuppression, and cancer treatment. World J. Gastroenterol. 2016, 22, 4794-4801. [CrossRef] [PubMed]

7. Chan, J.C.; Chan, D.L.; Diakos, C.I.; Engel, A.; Pavlakis, N.; Gill, A.; Clarke, S.J. The Lymphocyte-to-Monocyte Ratio is a Superior Predictor of Overall Survival in Comparison to Established Biomarkers of Resectable Colorectal Cancer. Ann. Surg. 2017, 265, 539-546. [CrossRef] [PubMed]

8. Li, M.X.; Liu, X.M.; Zhang, X.F.; Zhang, J.F.; Wang, W.L.; Zhu, Y.; Dong, J.; Cheng, J.W.; Liu, Z.W.; Ma, L.; et al. Prognostic role of neutrophil-to-lymphocyte ratio in colorectal cancer: A systematic review and meta-analysis. Int. J. Cancer 2014, 134, $2403-2413$. [CrossRef] [PubMed]

9. You, J.; Zhu, G.Q.; Xie, L.; Liu, W.Y.; Shi, L.; Wang, O.C.; Huang, Z.H.; Braddock, M.; Guo, G.L.; Zheng, M.H. Preoperative platelet to lymphocyte ratio is a valuable prognostic biomarker in patients with colorectal cancer. Oncotarget 2016, 7, 25516-25527. [CrossRef]

10. Song, Y.; Yang, Y.; Gao, P.; Chen, X.; Yu, D.; Xu, Y.; Zhao, J.; Wang, Z. The preoperative neutrophil to lymphocyte ratio is a superior indicator of prognosis compared with other inflammatory biomarkers in resectable colorectal cancer. BMC Cancer 2017, 17, 744 . [CrossRef]

11. Sipos, F.; Firneisz, G.; Muzes, G. Therapeutic aspects of c-MYC signaling in inflammatory and cancerous colonic diseases. World J. Gastroenterol. 2016, 22, 7938-7950. [CrossRef]

12. Terzic, J.; Grivennikov, S.; Karin, E.; Karin, M. Inflammation and colon cancer. Gastroenterology 2010, 138, 2101-2114 e2105. [CrossRef]

13. Ishii, M.; Wen, H.; Corsa, C.A.; Liu, T.; Coelho, A.L.; Allen, R.M.; Carson, W.F.t.; Cavassani, K.A.; Li, X.; Lukacs, N.W.; et al. Epigenetic regulation of the alternatively activated macrophage phenotype. Blood 2009, 114, 3244-3254. [CrossRef] [PubMed]

14. Saeed, S.; Quintin, J.; Kerstens, H.H.; Rao, N.A.; Aghajanirefah, A.; Matarese, F.; Cheng, S.C.; Ratter, J.; Berentsen, K.; van der Ent, M.A.; et al. Epigenetic programming of monocyte-to-macrophage differentiation and trained innate immunity. Science 2014, 345, 1251086. [CrossRef] [PubMed]

15. Jain, N.; Shahal, T.; Gabrieli, T.; Gilat, N.; Torchinsky, D.; Michaeli, Y.; Vogel, V.; Ebenstein, Y. Global modulation in DNA epigenetics during pro-inflammatory macrophage activation. Epigenetics 2019, 14, 1183-1193. [CrossRef]

16. Hanash, S. Integrated global profiling of cancer. Nat. Rev. Cancer 2004, 4, 638-644. [CrossRef]

17. Cancer Genome Atlas Research, N.; Weinstein, J.N.; Collisson, E.A.; Mills, G.B.; Shaw, K.R.; Ozenberger, B.A.; Ellrott, K.; Shmulevich, I.; Sander, C.; Stuart, J.M. The Cancer Genome Atlas Pan-Cancer analysis project. Nat. Genet. 2013, 45, 1113-1120. [CrossRef]

18. Cancer Genome Atlas Research, N. Comprehensive genomic characterization defines human glioblastoma genes and core pathways. Nature 2008, 455, 1061-1068. [CrossRef]

19. Kim, D.; Joung, J.G.; Sohn, K.A.; Shin, H.; Park, Y.R.; Ritchie, M.D.; Kim, J.H. Knowledge boosting: A graph-based integration approach with multi-omics data and genomic knowledge for cancer clinical outcome prediction. J. Am. Med. Inform. Assoc. 2015, 22, 109-120. [CrossRef]

20. Kristensen, V.N.; Lingjaerde, O.C.; Russnes, H.G.; Vollan, H.K.; Frigessi, A.; Borresen-Dale, A.L. Principles and methods of integrative genomic analyses in cancer. Nat. Rev. Cancer 2014, 14, 299-313. [CrossRef] [PubMed]

21. Kim, D.; Shin, H.; Song, Y.S.; Kim, J.H. Synergistic effect of different levels of genomic data for cancer clinical outcome prediction. J. Biomed. Inform. 2012, 45, 1191-1198. [CrossRef] [PubMed]

22. Kim, D.; Li, R.; Lucas, A.; Verma, S.S.; Dudek, S.M.; Ritchie, M.D. Using knowledge-driven genomic interactions for multi-omics data analysis: Metadimensional models for predicting clinical outcomes in ovarian carcinoma. J. Am. Med. Inform. Assoc. 2017, 24, 577-587. [CrossRef]

23. Brown, G.R.; Hem, V.; Katz, K.S.; Ovetsky, M.; Wallin, C.; Ermolaeva, O.; Tolstoy, I.; Tatusova, T.; Pruitt, K.D.; Maglott, D.R.; et al. Gene: A gene-centered information resource at NCBI. Nucleic Acids Res. 2015, 43, D36-D42. [CrossRef]

24. Harrell, F.E., Jr.; Lee, K.L.; Mark, D.B. Multivariable prognostic models: Issues in developing models, evaluating assumptions and adequacy, and measuring and reducing errors. Stat. Med. 1996, 15, 361-387. [CrossRef]

25. Hothorn, T.; Lausen, B. On the exact distribution of maximally selected rank statistics. J. Comput. Stat. Data Anal. 2003, 43, 121-137. [CrossRef] 
26. Wong, A.K.; Park, C.Y.; Greene, C.S.; Bongo, L.A.; Guan, Y.; Troyanskaya, O.G. IMP: A multi-species functional genomics portal for integration, visualization and prediction of protein functions and networks. Nucleic Acids Res. 2012, 40, W484-W490. [CrossRef] [PubMed]

27. Greene, C.S.; Krishnan, A.; Wong, A.K.; Ricciotti, E.; Zelaya, R.A.; Himmelstein, D.S.; Zhang, R.; Hartmann, B.M.; Zaslavsky, E.; Sealfon, S.C.; et al. Understanding multicellular function and disease with human tissue-specific networks. Nat. Genet. 2015, 47, 569-576. [CrossRef]

28. Oliveira, T.; Silva, A.; Satoh, K.; Julian, V.; Leao, P.; Novais, P. Survivability Prediction of Colorectal Cancer Patients: A System with Evolving Features for Continuous Improvement. Sensors 2018, 18, 2983. [CrossRef]

29. Gene [Internet]. Bethesda (MD): National Library of Medicine (US), National Center for Biotechnology Information. 2004. Available online: https:/ / www.ncbi.nlm.nih.gov/gene/ (accessed on 8 August 2019).

30. Li, J.; Lan, C.N.; Kong, Y.; Feng, S.S.; Huang, T. Identification and Analysis of Blood Gene Expression Signature for Osteoarthritis With Advanced Feature Selection Methods. Front. Genet. 2018, 9, 246. [CrossRef]

31. Jostins, L.; Ripke, S.; Weersma, R.K.; Duerr, R.H.; McGovern, D.P.; Hui, K.Y.; Lee, J.C.; Schumm, L.P.; Sharma, Y.; Anderson, C.A.; et al. Host-microbe interactions have shaped the genetic architecture of inflammatory bowel disease. Nature 2012, 491, 119-124. [CrossRef] [PubMed]

32. Gao, L.; Feng, Y.; Bowers, R.; Becker-Hapak, M.; Gardner, J.; Council, L.; Linette, G.; Zhao, H.; Cornelius, L.A. Ras-associated protein-1 regulates extracellular signal-regulated kinase activation and migration in melanoma cells: Two processes important to melanoma tumorigenesis and metastasis. Cancer Res. 2006, 66, 7880-7888. [CrossRef]

33. Zhang, L.; Chenwei, L.; Mahmood, R.; van Golen, K.; Greenson, J.; Li, G.; D’Silva, N.J.; Li, X.; Burant, C.F.; Logsdon, C.D.; et al. Identification of a putative tumor suppressor gene Rap1GAP in pancreatic cancer. Cancer Res. 2006, 66, 898-906. [CrossRef]

34. Li, P.; Wu, Y.H.; Zhu, Y.T.; Li, M.X.; Pei, H.H. Requirement of Rab21 in LPS-induced TLR4 signaling and pro-inflammatory responses in macrophages and monocytes. Biochem. Biophys. Res. Commun. 2019, 508, 169-176. [CrossRef] [PubMed]

35. Tang, B.L.; Ng, E.L. Rabs and cancer cell motility. Cell Motil. Cytoskeleton 2009, 66, 365-370. [CrossRef] [PubMed]

36. Hooper, S.; Gaggioli, C.; Sahai, E. A chemical biology screen reveals a role for Rab21-mediated control of actomyosin contractility in fibroblast-driven cancer invasion. Br. J. Cancer 2010, 102, 392-402. [CrossRef] [PubMed]

37. Araki, T.; Milbrandt, J. Ninjurin2, a novel homophilic adhesion molecule, is expressed in mature sensory and enteric neurons and promotes neurite outgrowth. J. Neurosci. 2000, 20, 187-195. [CrossRef] [PubMed]

38. Ifergan, I.; Kebir, H.; Terouz, S.; Alvarez, J.I.; Lecuyer, M.A.; Gendron, S.; Bourbonniere, L.; Dunay, I.R.; Bouthillier, A.; Moumdjian, R.; et al. Role of Ninjurin-1 in the migration of myeloid cells to central nervous system inflammatory lesions. Ann. Neurol. 2011, 70, 751-763. [CrossRef]

39. Cho, S.J.; Rossi, A.; Jung, Y.S.; Yan, W.; Liu, G.; Zhang, J.; Zhang, M.; Chen, X. Ninjurin1, a target of p53, regulates p53 expression and p53-dependent cell survival, senescence, and radiation-induced mortality. Proc. Natl. Acad. Sci. USA 2013, 110, 9362-9367. [CrossRef]

40. Langefeld, C.D.; Ainsworth, H.C.; Cunninghame Graham, D.S.; Kelly, J.A.; Comeau, M.E.; Marion, M.C.; Howard, T.D.; Ramos, P.S.; Croker, J.A.; Morris, D.L.; et al. Transancestral mapping and genetic load in systemic lupus erythematosus. Nat. Commun. 2017, 8, 16021. [CrossRef]

41. Stahl, E.A.; Raychaudhuri, S.; Remmers, E.F.; Xie, G.; Eyre, S.; Thomson, B.P.; Li, Y.; Kurreeman, F.A.; Zhernakova, A.; Hinks, A.; et al. Genome-wide association study meta-analysis identifies seven new rheumatoid arthritis risk loci. Nat. Genet. 2010, 42, 508-514. [CrossRef]

42. Komatsu, M.; Li, H.O.; Tsutsui, H.; Itakura, K.; Matsumura, M.; Yokoyama, K.K. MAZ, a Myc-associated zinc finger protein, is essential for the ME1a1-mediated expression of the c-myc gene during neuroectodermal differentiation of P19 cells. Oncogene 1997, 15, 1123-1131. [CrossRef] [PubMed]

43. Smith, D.R.; Goh, H.S. Overexpression of the c-myc proto-oncogene in colorectal carcinoma is associated with a reduced mortality that is abrogated by point mutation of the p53 tumor suppressor gene. Clin. Cancer Res. 1996, 2, 1049-1053.

44. Haberman, Y.; Karns, R.; Dexheimer, P.J.; Schirmer, M.; Somekh, J.; Jurickova, I.; Braun, T.; Novak, E.; Bauman, L.; Collins, M.H.; et al. Ulcerative colitis mucosal transcriptomes reveal mitochondriopathy and personalized mechanisms underlying disease severity and treatment response. Nat. Commun. 2019, 10, 38. [CrossRef]

45. Ahechu, P.; Zozaya, G.; Marti, P.; Hernandez-Lizoain, J.L.; Baixauli, J.; Unamuno, X.; Fruhbeck, G.; Catalan, V. NLRP3 Inflammasome: A Possible Link Between Obesity-Associated Low-Grade Chronic Inflammation and Colorectal Cancer Development. Front. Immunol. 2018, 9, 2918. [CrossRef]

46. Seo, T.; Tatsuguchi, A.; Shinji, S.; Yonezawa, M.; Mitsui, K.; Tanaka, S.; Fujimori, S.; Gudis, K.; Fukuda, Y.; Sakamoto, C. Microsomal prostaglandin E synthase protein levels correlate with prognosis in colorectal cancer patients. Virchows Arch. 2009, 454, 667-676. [CrossRef]

47. Yoshimatsu, K.; Golijanin, D.; Paty, P.B.; Soslow, R.A.; Jakobsson, P.J.; DeLellis, R.A.; Subbaramaiah, K.; Dannenberg, A.J. Inducible microsomal prostaglandin E synthase is overexpressed in colorectal adenomas and cancer. Clin. Cancer Res. 2001, 7, 3971-3976. [PubMed]

48. Williams, A.D.; Korolkova, O.Y.; Sakwe, A.M.; Geiger, T.M.; James, S.D.; Muldoon, R.L.; Herline, A.J.; Goodwin, J.S.; Izban, M.G.; Washington, M.K.; et al. Human alpha defensin 5 is a candidate biomarker to delineate inflammatory bowel disease. PLoS ONE 2017, 12, e0179710. [CrossRef] 
49. Alquraini, A.; Jamal, M.; Zhang, L.; Schmidt, T.; Jay, G.D.; Elsaid, K.A. The autocrine role of proteoglycan-4 (PRG4) in modulating osteoarthritic synoviocyte proliferation and expression of matrix degrading enzymes. Arthritis Res. Ther. 2017, 19, 89. [CrossRef]

50. Iqbal, S.M.; Leonard, C.; Regmi, S.C.; De Rantere, D.; Tailor, P.; Ren, G.; Ishida, H.; Hsu, C.; Abubacker, S.; Pang, D.S.; et al. Lubricin/Proteoglycan 4 binds to and regulates the activity of Toll-Like Receptors In Vitro. Sci. Rep. 2016, 6, 18910. [CrossRef]

51. Sarkar, A.; Chanda, A.; Regmi, S.C.; Karve, K.; Deng, L.; Jay, G.D.; Jirik, F.R.; Schmidt, T.A.; Bonni, S. Recombinant human PRG4 (rhPRG4) suppresses breast cancer cell invasion by inhibiting TGFbeta-Hyaluronan-CD44 signalling pathway. PLoS ONE 2019, 14, e0219697. [CrossRef]

52. Farwell, S.L.; Kanyi, D.; Hamel, M.; Slee, J.B.; Miller, E.A.; Cipolle, M.D.; Lowe-Krentz, L.J. Heparin Decreases in Tumor Necrosis Factor alpha (TNFalpha)-induced Endothelial Stress Responses Require Transmembrane Protein 184A and Induction of Dual Specificity Phosphatase 1. J. Biol. Chem. 2016, 291, 5342-5354. [CrossRef] [PubMed]

53. Surveillance, Epidemiology, and End Results (SEER) Program SEER *Stat Database: Incidence-SEER 18 Regs Public-Use. October 2010 Sub (1973-2008 Varying); National Cancer Institute, DCCPS, Surveillance Research Program, Cancer Statistics Branch, US, 2011. Available online: https:/ / seer.cancer.gov (accessed on 14 December 2020).

54. Hari, D.M.; Leung, A.M.; Lee, J.H.; Sim, M.S.; Vuong, B.; Chiu, C.G.; Bilchik, A.J. AJCC Cancer Staging Manual 7th edition criteria for colon cancer: Do the complex modifications improve prognostic assessment? J. Am. Coll. Surg. 2013, 217, 181-190. [CrossRef] [PubMed] 